\title{
A retroperitoneal mass, systemic lymphadenopathy, and pulmonary nodules in a pregnant woman
}

\author{
Koichi Ogura • Ako Hosono • Akihiko Yoshida • \\ Yasuo Beppu • Akira Kawai
}

Received: 3 August 2010 /Revised: 15 December 2010 /Accepted: 22 December 2010/Published online: 1 February 2011

(C) ISS 2011

\begin{abstract}
Answer
Diagnosis

Lymphangioleiomyomatosis (LAM).

Discussion

Lymphangioleiomyomatosis is a rare disease characterized by abnormal proliferation of smooth muscle-like cells that exhibit myomelanotic differentiation represented by actin and HMB45 coexpression. It develops almost exclusively in women of reproductive age $[1,2]$. Although the precise incidence and prevalence of LAM remain unknown, this disorder probably represents less than $1 \%$ of all diffuse lung diseases [3].

In the vast majority of cases, the initial manifestations of LAM are pulmonary symptoms including dyspnea,
\end{abstract}

The case presentation can be found at doi:10.1007/s00256-010-10911.

K. Ogura $\cdot$ Y. Beppu $\cdot$ A. Kawai $(\bowtie)$

Division of Musculoskeletal Oncology,

National Cancer Center Hospital,

5-1-1 Tsukiji, Chuo-ku,

Tokyo 104-0045, Japan

e-mail: akawai@ncc.go.jp

\section{A. Hosono}

Division of Paediatric Oncology,

National Cancer Center Hospital,

5-1-1 Tsukiji, Chuo-ku,

Tokyo 104-0045, Japan

\section{A. Yoshida}

Division of Pathology, National Cancer Center Hospital,

5-1-1 Tsukiji, Chuo-ku,

Tokyo 104-0045, Japan chest pain, recurrent spontaneous pneumothorax, or chylous pleural effusion [4]. The diagnosis of LAM is usually made based on the above-mentioned typical clinical features and imaging characteristics of diffuse bilateral thin-walled cysts [5]. Extrapulmonary LAM involvement is uncommonly seen as a minor component associated with typical pulmonary lesions. The case reported here is unusual in that LAM manifested initially as massive extrapulmonary involvement including superficial lymphadenopathy, and the large retroperitoneal mass with diffuse nodal spread closely simulating malignant neoplasms such as lymphoma, gynecological malignancy, or retroperitoneal soft tissue sarcoma. In addition, small pulmonary nodules, not typical of LAM [5], complicated the picture and raised the possibility of widespread pulmonary metastases.

Extrapulmonary LAM mostly affects three major locations: the posterior mediastinum, the upper retroperitoneal areas close to the abdominal aorta, and the pelvic cavity [6]. This unique tendency is due to the fact that these sites are particularly rich in lymphatic vessels and lymph nodes, and LAM cells have a peculiar proclivity to proliferate around the lymphatics and migrate along these structures. Supraclavicular involvement observed in this case is exceptional.

Estrogen and progesterone are considered to play a potential etiological role in LAM development. This is primarily suggested by the fact that LAM most commonly presents in women of reproductive age [1, 2]. In addition, pregnancy or estrogen administration is reported to aggravate LAM [7], and patients are frequently advised to avoid pregnancy [7, 8]. LAM cells are reported to express estrogen and/or progesterone receptors in half of the cases, and hypersecretion of sex hormones during pregnancy may trigger overstimulation 
and proliferation of lesional cells. It is then tempting to speculate that the presence of nuclear atypia and mitotic activity in the present case, unusual findings for LAM, may have reflected modulation of the hormonal environment due to the twin pregnancy. The dramatic postpartum radiological improvement seen in the patient also highlights the close link between LAM and sex hormone activity.

This case of LAM, which manifested predominantly as extensive extrapulmonary disease without significant pulmonary symptoms, emphasizes the need for LAM to be included in the differential diagnosis of any mass lesion in the internal trunk of a young woman. A retroperitoneal mass with lymphatic spread is generally considered to be a sign of malignancy, and LAM patients with such presentation may be referred to orthopaedic oncologists who may not be familiar with this condition. Recognizing the mass-forming variant of LAM is important, in order to provide appropriate management and to avoid unnecessary cytotoxic or surgical treatment.

\section{References}

1. Johnson SR, Tattersfield AE. Clinical experience of lymphangioleiomyomatosis in the UK. Thorax. 2000;55(12):1052-7.

2. Ryu JH, Moss J, Beck GJ, Lee JC, Brown KK, Chapman JT, et al. The NHLBI lymphangioleiomyomatosis registry: characteristics of 230 patients at enrollment. Am J Respir Crit Care Med. 2006;173(1):105-11.

3. Lam B, Ooi GC, Wong MP, Lee R, Ip MS, Chan-Yeung M, et al. Extrapulmonary presentation of asymptomatic pulmonary lymphangioleiomyomatosis. Respirology. 2003;8(4):544-7.

4. Chu SC, Horiba K, Usuki J, Avila NA, Chen CC, Travis WD, et al. Comprehensive evaluation of 35 patients with lymphangioleiomyomatosis. Chest. 1999;115(4):1041-52.

5. Abbott GF, Rosado-de-Christenson ML, Frazier AA, Franks TJ, Pugatch RD, Galvin JR. From the archives of the AFIP: lymphangioleiomyomatosis: radiologic-pathologic correlation. Radiographics. 2005;25(3):803-28.

6. Matsui K, Tatsuguchi A, Valencia J, Yu Z, Bechtle J, Beasley MB, et al. Extrapulmonary lymphangioleiomyomatosis (LAM): clinicopathologic features in 22 cases. Hum Pathol. 2000;31(10):1242-8.

7. Brunelli A, Catalini G, Fianchini A. Pregnancy exacerbating unsuspected mediastinal lymphangioleiomyomatosis and chylothorax. Int J Gynaecol Obstet. 1996;52(3):289-90.

8. Cohen MM, Freyer AM, Johnson SR. Pregnancy experiences among women with lymphangioleiomyomatosis. Respir Med. 2009;103(5):766-72. 